\title{
Evaluation of Heavy Metal Concentrations in Soil and Edible Vegetables Grown in Compost from Unknown Sources in Al-Jiftlik, Palestine
}

\author{
Mohammed Bawwab', Ahmad Qutob2, Mahmoud Al Khatib33, Husam Malassa ${ }^{3}$, \\ Ayman Shawahna ${ }^{4}$, Mutaz Qutob ${ }^{3 *}$

\footnotetext{
${ }^{1}$ Department of Materials Engineering, Faculty of Engineering, Al-Quds University, Jerusalem, Palestine ${ }^{2}$ Department of Electrical Engineering, Faculty of Engineering, Al-Quds University, Jerusalem, Palestine ${ }^{3}$ Department of Chemistry and Chemical Technology, Al-Quds University, Jerusalem, Palestine, ${ }^{4}$ Environment Quality Authority, Al-Bireh, Palestine

Email: ^kutob@staff.alquds.edu
}

How to cite this paper: Bawwab, M., Qutob, A., Al Khatib, M., Malassa, H., Shawahna, A. and Qutob, M. (2022) Evaluation of Heavy Metal Concentrations in Soil and Edible Vegetables Grown in Compost from Unknown Sources in Al-Jiftlik, Palestine. Journal of Environmental Protection, 13, 112-125. https://doi.org/10.4236/jep.2022.131007

Received: November 4, 2021

Accepted: January 14, 2022

Published: January 17, 2022

Copyright $\odot 2022$ by author(s) and Scientific Research Publishing Inc. This work is licensed under the Creative Commons Attribution International License (CC BY 4.0).

http://creativecommons.org/licenses/by/4.0/

\begin{abstract}
The use of compost as a fertilizer has been widely used in many countries. However, compost that contains heavy metals can transfer these metals to soils and plants (vegetables). This study investigates the concentrations of metals in soil and edible vegetables that were fertilized by polluted unknown compost in Al-Jiftlik region (Palestine). The source of the compost is the autocratic dumping sites of the Israeli settlements. The compost is distributed free of charge to the Palestinian farmers. The concentrations of $\mathrm{Ba}, \mathrm{Cu}$, $\mathrm{Pb}, \mathrm{Th}, \mathrm{Se}, \mathrm{Mn}, \mathrm{Co}$ and As in the contaminated farms were measured. Vegetables include: Eggplant, Corn, Bell Pepper, Cucumber and Marrow. Metals availability as well as the $\mathrm{pH}$, was also examined in the soil samples. Normal farms that did not use this unknown compost were used as a reference. The concentrations of $\mathrm{Ba}, \mathrm{Cu}, \mathrm{Pb}, \mathrm{Th}, \mathrm{Se}, \mathrm{Mn}, \mathrm{Co}$ and $\mathrm{As}$ in soil and vegetables in the polluted farms were above the WHO limits. Barium concentrations in the vegetables were ranged from $1.00 \mathrm{mg} / \mathrm{kg}$ to $0.453 \mathrm{mg} / \mathrm{kg}$. It is high when compared to WHO limit of $0.3 \mathrm{mg} / \mathrm{kg}$. Copper concentrations in the vegetables were ranged from $63.84 \mathrm{mg} / \mathrm{kg}$ to $50.53 \mathrm{mg} / \mathrm{kg}$. It is high when compared to WHO limit of $40 \mathrm{mg} / \mathrm{kg}$. Lead concentrations in the vegetables were ranged from $1.00 \mathrm{mg} / \mathrm{kg}$ to $0.453 \mathrm{mg} / \mathrm{kg}$. Lead concentration is high when compared to WHO limit of $0.3 \mathrm{mg} / \mathrm{kg}$. Thallium concentrations in the vegetables were ranged from $2.99 \mathrm{mg} / \mathrm{kg}$ to $1.22 \mathrm{mg} / \mathrm{kg}$. Thallium concentration is high when compared to WHO limit of $0.3 \mathrm{mg} / \mathrm{kg}$. Selenium concentrations in the vegetables were ranged from $0.550 \mathrm{mg} / \mathrm{kg}$ to $0.348 \mathrm{mg} / \mathrm{kg}$. It is high when
\end{abstract}


compared to WHO limit of $0.3 \mathrm{mg} / \mathrm{kg}$. Manganese concentrations in the vegetables were ranged from $825.3 \mathrm{mg} / \mathrm{kg}$ to $446.2 \mathrm{mg} / \mathrm{kg}$. It is almost high when compared to WHO limit of $500 \mathrm{mg} / \mathrm{kg}$. Cobalt concentrations in the vegetables were ranged from $1.119 \mathrm{mg} / \mathrm{kg}$ to $0.522 \mathrm{mg} / \mathrm{kg}$. Cobalt concentration is high when compared to WHO limit of $0.1 \mathrm{mg} / \mathrm{kg}$. Arsenic concentrations in the vegetables were ranged from $4.306 \mathrm{mg} / \mathrm{kg}$ to $0.662 \mathrm{mg} / \mathrm{kg}$. It is high when compared to WHO limit of $0.2 \mathrm{mg} / \mathrm{kg}$. On the other hand, all metals concentrations in the clean farms were below the WHO limits. The study had recommended preventing farmers from using this unknown compost.

\section{Keywords}

Compost, Heavy Metals, Vegetables, Autocratic Dumping

\section{Introduction}

Food quality and safety are global issues [1]. Agricultural products free of chemical contaminants especially heavy metals are important for food safety [1] [2]. Consumption of food products contaminated with metals (repeated) may cause health risks to people. They are often considered to be toxic-and toxicity depends on the dose of the toxic metal, route of exposure, and the nutritional status of the exposed human being [3] [4]. The dangerous effects of heavy metals lie in the fact that it tends to be bio-accumulated in different organs or tissues. The bioaccumulation of these compounds in living things is due to their speed of taking [5].

Compost is a decomposed organic fertilizer resulting from the fermentation and decomposition of chopped plant residues, animal waste or aerobic garbage [6]. The use of compost as a fertilizer is significant enthusiasm for the soil and for keeping up an appropriate soil structure as well as adding organic matter that is lost due to the practice of intensive agriculture [6] [7]. Among the possible negative impacts of applying compost to cropland, are the potential arrival of dangerous metals into the soil, and the exchange of these components from the soil to the food cycle [8]. In order to use compost as a fertilizer it should be free from any Plastic materials, metals and metal objects, Grease, petroleum materials and contaminated materials [8] [9].

Recently, some regions in the West Bank agricultural land have been used as random dumps and landfills for Israeli solid wastes, especially the toxic wastes [10] [11]. Most of these lands are located close to Israeli settlements and beyond the separation wall. This operation is often carried out in coordination with local contractors. The unknown waste, which is transported from the Israeli settlements, is buried in nearby agricultural lands. The dumping waste is not subjected to any waste treatment process or any supervision practice according to international standards [12] The process of environmental pollution that begins with the soil, water, unpleasant smells and bad appearance continue by distri- 
buting freely without any charge the compost to the Palestinian farmer in order to empty the landfill for future dumping.

This study investigates the concentrations of metals in soil that was fertilized by polluted unknown compost in Al-Jiftlik region. The study also investigate the concentrations of metals in vegetable crops that were grown on this compost and evaluate their contamination level with respect to food standard guidelines. Vegetables include: Eggplant, Corn, Bell Pepper, Cucumber and Marrow.

Barium, Copper, lead, Thallium, Selenium, Manganese, Cobalt and Arsenic, metals were measured in soil and vegetables. The results were compared to International standards (FAO/WHO) [13]. In addition, $\mathrm{pH}$ of the polluted soil was measured and analyzed. The $\mathrm{pH}$ value has a direct effect on the soil. It is related to soil properties as cation exchange Capacity, particle size distribution, organic matter content and oxide content. These characteristics have a direct effect on heavy metal accumulation and affect their transfer to crops, vegetables, leaves or other components of the environment [14].

This study has significant importance to locals who consume these vegetables, farmers who plant these vegetables, legislators and relevant fields.

\section{Experimental}

\subsection{Study Area}

Al Jiftlik is a Palestinian village in Jericho governorate in the west bank. The village is located (horizontally) $33 \mathrm{~km}$ north of Jericho city, its land area is 1242 dunams. The village crosses the main street in the middle and divides it in two halves east and west, latitude of $32^{\circ} 08^{\prime} 39^{\prime \prime} \mathrm{N}$ longitude of $35^{\circ} 29^{\prime} 36^{\prime \prime} \mathrm{E}$ [15] (Figure 1). It is located on the border of the Jordan Valley in the occupied Palestinian territories. The village is bordered by the Jordan River to the East, Marj Al Ghazal village and Tubas Governorate lands to the north. The surrounding villages of Nablus Governorate to the east include Duma, Majdal Bani Fadil, Aqraba to the west and Al Fasayil village to the south. Few kilometers away from Jiftlik are Israeli settlements [15].

\subsection{Climate}

The area has an arid Mediterranean climate. The mean annual rainfall is 232 $\mathrm{mm}$. The average annual temperature is $22^{\circ} \mathrm{C}$, and the average annual humidity is approximately $49.2 \%[15]$.

\subsection{Soil Types}

Desert alluvial soil was formed as a result of erosion of calcareous silty and clayey materials. This soil type supports herbaceous vegetation of desert annual halophytes and glycophytes and responds well to irrigation, producing various crops, mainly subtropical and tropical fruits, such as citrus, bananas, and dates, as well as winter vegetables. The American great group classifications that represent this soil association are Haplargids and Camborthids [10] [11]. 


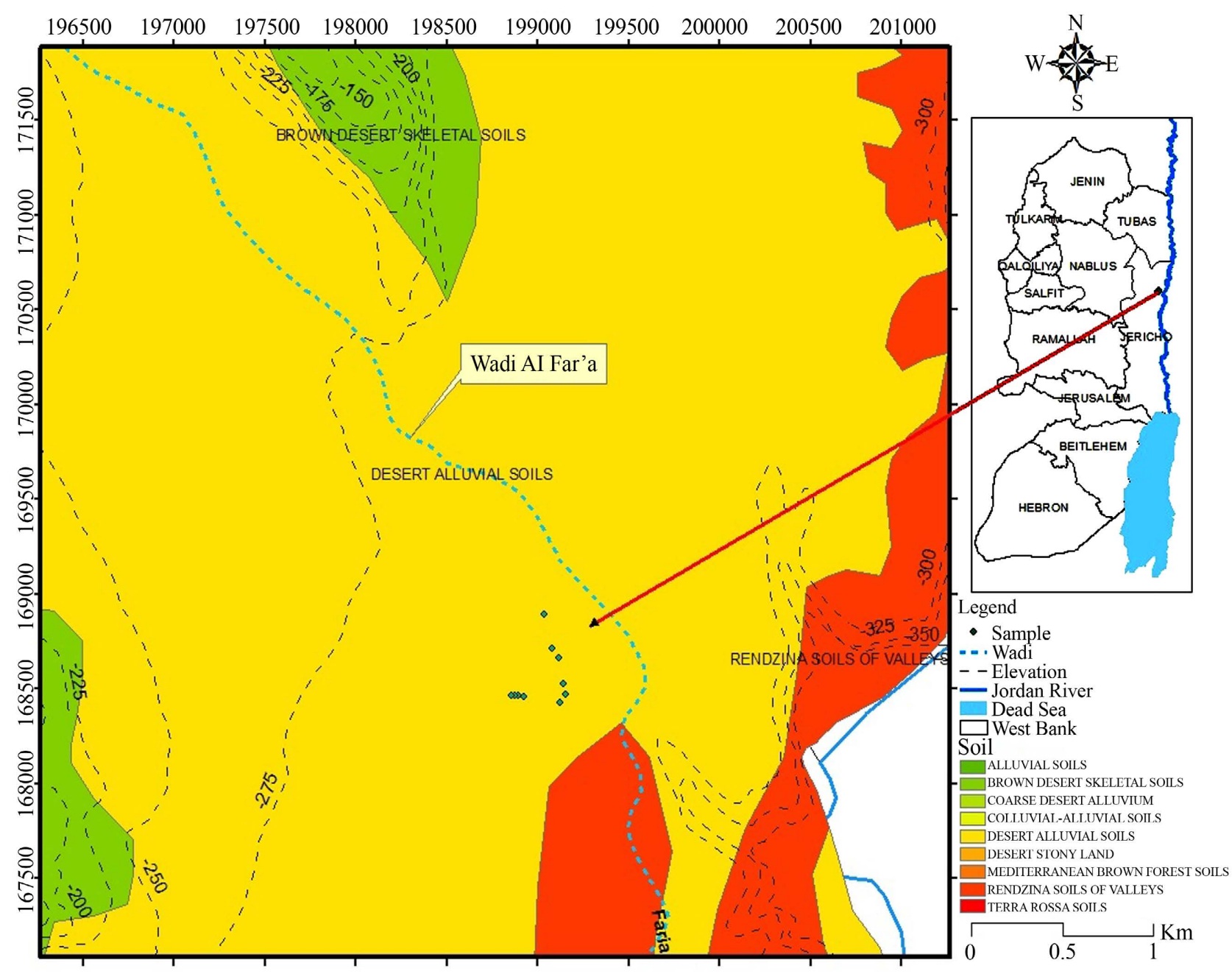

Figure 1. Map of West Bank and topography of the study area Al Jiftlik. Source: GIS laboratory at Al-Quds University. Locations of the sampling sites are indicated.

\subsection{Population}

According to the Palestinian Central Bureau of Statistics (PCBS), the total population of $\mathrm{Al}$ Jiftlik in 2007 was 3714; of whom 1857 were male and 1857, female. There were additionally registered to be 578 households living in 692 housing units [16].

\subsection{Economy}

The economy in Al Jiftlik depends on several economic sectors, mainly: the agriculture sector, which absorbs $90 \%$ of the human workforce. The results of a field survey conducted by ARIJ for the distribution of labor by economic activity Al Jiftlik are as follows [15]:
1) Agriculture Sector (90\%);
2) Trade Sector (5\%);
3) Israeli Labor Market (3\%);
4) Government or Private Employees Sector (2\%). 


\section{Sampling and Analysis}

\subsection{Soil Sampling}

20 soil samples were sampled from both polluted and reference clean locations. Samples were taken from the surface and from the depth of $0-30 \mathrm{~cm}$ according to the type of vegetables. The coordination and the vegetable type are shown in Table 1. Sampling was done according to the random method [17] for each location as shown in Figure 2. The sampling method includes:

1) A sample from each location (polluted, Reference) was taken from soil, water and vegetables. Vegetables include: Eggplant, Corn, Bell Pepper, Cucumber and Marrow.

2) The representative sample was taken by the identification of circle with a radius of 6 meter (Figure 2).

3) Five cores were sampled inside the 6-meter circle at the surface and at a depth of $0-30 \mathrm{~cm}$ from all the sites (Figure 2).

4) The five cores were mixed with each other to form a homogeneous represented sample according to each depth, site (polluted, Reference) and the type of vegetables.

5) The representative samples were analyzed in the laboratory.

\subsection{Vegetables Sampling}

10 representative vegetable samples were taken during November-December 2019, from contaminated and reference vegetables. In addition, in April 2018, three more vegetable samples (Marrow, Eggplant, Bell pepper) were sampled from the same sites.

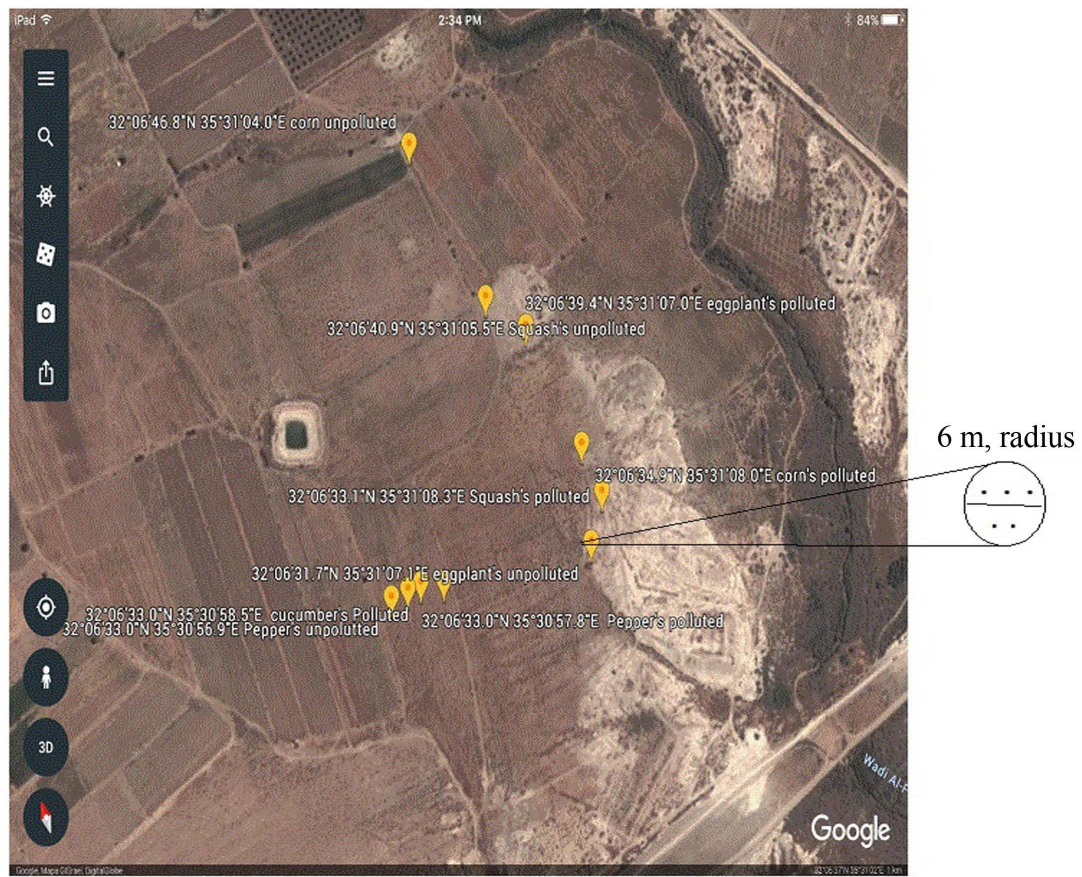

Figure 2. Sampling of polluted and reference soil samples (Google earth, 2019). 
Table 1. Heavy metals concentration in soil samples.

\begin{tabular}{|c|c|c|c|c|c|c|c|c|}
\hline \multirow{2}{*}{ Soil samples and depth } & \multicolumn{8}{|c|}{ Metals Concentration $(\mathrm{mg} / \mathrm{kg})$} \\
\hline & $\mathrm{Ba}$ & $\mathrm{Tl}$ & Mn & Co & $\mathrm{Cu}$ & As & $\mathrm{Se}$ & $\mathrm{Pb}$ \\
\hline $\begin{array}{l}\text { Polluted soil from Corn farms, } \\
\qquad 0 \mathrm{~cm}\end{array}$ & $\begin{array}{c}175.962 \\
\pm \\
0.003\end{array}$ & $\begin{array}{c}11.926 \\
\pm \\
0.182\end{array}$ & $\begin{array}{c}741.838 \\
\pm \\
0.003\end{array}$ & $\begin{array}{c}17.934 \\
\pm \\
0.006\end{array}$ & $\begin{array}{c}17.656 \\
\pm \\
0.026\end{array}$ & $\begin{array}{c}2.384 \\
\pm \\
0.014\end{array}$ & $\begin{array}{c}5.860 \\
\pm \\
0.133\end{array}$ & $\begin{array}{c}12.211 \\
\pm \\
0.041\end{array}$ \\
\hline $\begin{array}{l}\text { Polluted soil from Corn farms, } \\
\qquad 0-30 \mathrm{~cm}\end{array}$ & $\begin{array}{c}230.295 \\
\pm \\
0.004\end{array}$ & $\begin{array}{c}12.910 \\
\pm \\
0.019\end{array}$ & $\begin{array}{c}1075.468 \\
\pm \\
0.004\end{array}$ & $\begin{array}{c}27.315 \\
\pm \\
0.005\end{array}$ & $\begin{array}{c}34.870 \\
\pm \\
0.019\end{array}$ & $\begin{array}{c}3.360 \\
\pm \\
0.016\end{array}$ & $\begin{array}{c}10.497 \\
\pm \\
0.101\end{array}$ & $\begin{array}{c}24.106 \\
\pm \\
0.010\end{array}$ \\
\hline $\begin{array}{l}\text { Clean soil from Corn farms, } \\
\qquad 0 \mathrm{~cm}\end{array}$ & $\begin{array}{c}15.043 \\
\pm \\
0.001\end{array}$ & $\begin{array}{c}2.320 \\
\pm \\
0.614\end{array}$ & $\begin{array}{c}128.587 \\
\pm \\
0.004\end{array}$ & $\begin{array}{c}4.514 \\
\pm \\
0.015\end{array}$ & $\begin{array}{c}0.141 \\
\pm \\
0.013\end{array}$ & $\begin{array}{c}0.017 \\
\pm \\
0.044\end{array}$ & $\begin{array}{c}0.051 \\
\pm \\
0.170\end{array}$ & $\begin{array}{c}4.020 \\
\pm \\
0.001\end{array}$ \\
\hline $\begin{array}{l}\text { Clean soil from Corn farms, } \\
\qquad 0-30 \mathrm{~cm}\end{array}$ & $\begin{array}{c}12.067 \\
\pm \\
0.006\end{array}$ & $\begin{array}{c}2.144 \\
\pm \\
0.022\end{array}$ & $\begin{array}{c}94.324 \\
\pm \\
0.011\end{array}$ & $\begin{array}{c}2.254 \\
\pm \\
0.020\end{array}$ & $\begin{array}{c}0.133 \\
\pm \\
0.009\end{array}$ & $\begin{array}{c}0.012 \\
\pm \\
0.047\end{array}$ & $\begin{array}{c}0.064 \\
\pm \\
0.095\end{array}$ & $\begin{array}{c}2.955 \\
\pm \\
0.041\end{array}$ \\
\hline $\begin{array}{l}\text { Polluted soil from Bell pepper farms, } \\
\qquad 0 \mathrm{~cm}\end{array}$ & $\begin{array}{c}151.793 \\
\pm \\
0.002\end{array}$ & $\begin{array}{c}22.042 \\
\pm \\
0.786\end{array}$ & $\begin{array}{c}951.016 \\
\pm \\
0.004\end{array}$ & $\begin{array}{c}19.056 \\
\pm \\
0.011\end{array}$ & $\begin{array}{c}12.342 \\
\pm \\
0.077\end{array}$ & $\begin{array}{c}5.172 \\
\pm \\
0.026\end{array}$ & $\begin{array}{c}12.871 \\
\pm \\
0.045\end{array}$ & $\begin{array}{c}34.144 \\
\pm \\
0.065\end{array}$ \\
\hline $\begin{array}{l}\text { Polluted soil from Bell pepper farms, } \\
\qquad 0-30 \mathrm{~cm}\end{array}$ & $\begin{array}{c}136.639 \\
\pm \\
0.004\end{array}$ & $\begin{array}{c}11.600 \\
\pm \\
0.003\end{array}$ & $\begin{array}{c}744.514 \\
\pm \\
0.002\end{array}$ & $\begin{array}{c}12.197 \\
\pm \\
0.004\end{array}$ & $\begin{array}{c}10.112 \\
\pm \\
0.050\end{array}$ & $\begin{array}{c}5.237 \\
\pm \\
0.017\end{array}$ & $\begin{array}{c}10.536 \\
\pm \\
0.092\end{array}$ & $\begin{array}{c}22.915 \\
\pm \\
0.019\end{array}$ \\
\hline $\begin{array}{l}\text { Clean soil from Bell pepper farms, } \\
\qquad 0 \mathrm{~cm}\end{array}$ & $\begin{array}{c}14.060 \\
\pm \\
0.004\end{array}$ & $\begin{array}{c}1.234 \\
\pm \\
0.098\end{array}$ & $\begin{array}{c}109.044 \\
\pm \\
0.004\end{array}$ & $\begin{array}{c}3.670 \\
\pm \\
0.013\end{array}$ & $\begin{array}{c}0.082 \\
\pm \\
0.011\end{array}$ & $\begin{array}{c}0.007 \\
\pm \\
0.024\end{array}$ & $\begin{array}{c}0.311 \\
\pm \\
0.027\end{array}$ & $\begin{array}{c}5.344 \\
\pm \\
0.002\end{array}$ \\
\hline $\begin{array}{l}\text { Clean soil from Bell pepper farms, } \\
\qquad 0-30 \mathrm{~cm}\end{array}$ & $\begin{array}{c}7.848 \\
\pm \\
0.003\end{array}$ & $\begin{array}{c}0.168 \\
\pm \\
0.057\end{array}$ & $\begin{array}{c}109.044 \\
\pm \\
0.001\end{array}$ & $\begin{array}{c}0.369 \\
\pm \\
0.046\end{array}$ & $\begin{array}{c}0.021 \\
\pm \\
0.004\end{array}$ & $\begin{array}{c}0.007 \\
\pm \\
0.030\end{array}$ & $\begin{array}{c}0.066 \\
\pm \\
0.036\end{array}$ & $\begin{array}{c}5.360 \\
\pm \\
0.002\end{array}$ \\
\hline $\begin{array}{l}\text { Polluted soil from Eggplant farms, } \\
\qquad 0 \mathrm{~cm}\end{array}$ & $\begin{array}{c}125.384 \\
\pm \\
0.003\end{array}$ & $\begin{array}{c}23.200 \\
\pm \\
0.156\end{array}$ & $\begin{array}{c}478.976 \\
\pm \\
0.003\end{array}$ & $\begin{array}{c}26.062 \\
\pm \\
0.009\end{array}$ & $\begin{array}{c}13.709 \\
\pm \\
0.006\end{array}$ & $\begin{array}{c}6.362 \\
\pm \\
0.024\end{array}$ & $\begin{array}{c}3.621 \\
\pm \\
0.218\end{array}$ & $\begin{array}{c}12.651 \\
\pm \\
0.013\end{array}$ \\
\hline $\begin{array}{l}\text { Polluted soil from Eggplant farms, } \\
\qquad 0-30 \mathrm{~cm}\end{array}$ & $\begin{array}{c}62.641 \\
\pm \\
0.006\end{array}$ & $\begin{array}{c}16.020 \\
\pm \\
0.056\end{array}$ & $\begin{array}{c}273.810 \\
\pm \\
0.005\end{array}$ & $\begin{array}{l}14.655 \\
\pm \\
0.005\end{array}$ & $\begin{array}{l}7.110 \\
\pm \\
0.019\end{array}$ & $\begin{array}{l}3.264 \\
\pm \\
0.027\end{array}$ & $\begin{array}{l}2.781 \\
\pm \\
0.772\end{array}$ & $\begin{array}{c}10.216 \\
\pm \\
0.051\end{array}$ \\
\hline $\begin{array}{l}\text { Clean soil from Eggplant farms, } \\
0 \mathrm{~cm}\end{array}$ & $\begin{array}{c}18.050 \\
\pm \\
0.002\end{array}$ & $\begin{array}{c}1.624 \\
\pm \\
0.020\end{array}$ & $\begin{array}{l}73.053 \\
\pm \\
0.009\end{array}$ & $\begin{array}{c}3.931 \\
\pm \\
0.005\end{array}$ & $\begin{array}{c}0.111 \\
\pm \\
0.014\end{array}$ & $\begin{array}{c}0.018 \\
\pm \\
0.016\end{array}$ & $\begin{array}{c}0.406 \\
\pm \\
0.038\end{array}$ & $\begin{array}{l}2.680 \\
\pm \\
0.020\end{array}$ \\
\hline $\begin{array}{l}\text { Clean soil from Eggplant farms, } \\
\qquad 0-30 \mathrm{~cm}\end{array}$ & $\begin{array}{c}34.028 \\
\pm \\
0.006\end{array}$ & $\begin{array}{c}2.320 \\
\pm \\
0.001\end{array}$ & $\begin{array}{c}145.392 \\
\pm \\
0.002\end{array}$ & $\begin{array}{c}0.100 \\
\pm \\
0.003\end{array}$ & $\begin{array}{c}0.031 \\
\pm \\
0.012\end{array}$ & $\begin{array}{c}0.006 \\
\pm \\
0.055\end{array}$ & $\begin{array}{c}0.031 \\
\pm \\
0.121\end{array}$ & $\begin{array}{c}3.350 \\
\pm \\
0.024\end{array}$ \\
\hline $\begin{array}{l}\text { Polluted soil from Cucumber farms, } \\
\qquad 0 \mathrm{~cm}\end{array}$ & $\begin{array}{c}103.193 \\
\pm \\
0.005\end{array}$ & $\begin{array}{c}24.932 \\
\pm \\
0.032\end{array}$ & $\begin{array}{c}572.315 \\
\pm \\
0.002\end{array}$ & $\begin{array}{c}11.019 \\
\pm \\
0.008\end{array}$ & $\begin{array}{c}10.766 \\
\pm \\
0.173\end{array}$ & $\begin{array}{c}3.423 \\
\pm \\
0.026\end{array}$ & $\begin{array}{c}7.600 \\
\pm \\
0.067\end{array}$ & $\begin{array}{c}21.310 \\
\pm \\
0.022\end{array}$ \\
\hline $\begin{array}{l}\text { Polluted soil from Cucumber farms, } \\
\qquad 0-30 \mathrm{~cm}\end{array}$ & $\begin{array}{c}70.178 \\
\pm \\
0.005\end{array}$ & $\begin{array}{c}14.243 \\
\pm \\
0.028\end{array}$ & $\begin{array}{c}545.220 \\
\pm \\
0.002\end{array}$ & $\begin{array}{c}2.383 \\
\pm \\
0.017\end{array}$ & $\begin{array}{c}9.000 \\
\pm \\
0.016\end{array}$ & $\begin{array}{c}1.573 \\
\pm \\
0.019\end{array}$ & $\begin{array}{c}5.056 \\
\pm \\
0.150\end{array}$ & $\begin{array}{c}14.402 \\
\pm \\
0.038\end{array}$ \\
\hline
\end{tabular}




\section{Continued}

\begin{tabular}{|c|c|c|c|c|c|c|c|c|}
\hline $\begin{array}{c}\text { Clean soil from Cucumber farms, } \\
0 \mathrm{~cm}\end{array}$ & $\begin{array}{c}7.673 \\
\pm \\
0.003\end{array}$ & $\begin{array}{c}1.624 \\
\pm \\
0.960\end{array}$ & $\begin{array}{c}117.524 \\
\pm \\
0.081\end{array}$ & $\begin{array}{c}4.627 \\
\pm \\
0.009\end{array}$ & $\begin{array}{c}0.149 \\
\pm \\
0.009\end{array}$ & $\begin{array}{c}0.010 \\
\pm \\
0.022\end{array}$ & $\begin{array}{c}0.082 \\
\pm \\
0.028\end{array}$ & $\begin{array}{c}4.262 \\
\pm \\
0.022\end{array}$ \\
\hline $\begin{array}{l}\text { Clean soil from Cucumber farms, } \\
\qquad 0-30 \mathrm{~cm}\end{array}$ & $\begin{array}{c}9.124 \\
\pm \\
0.010\end{array}$ & $\begin{array}{c}2.320 \\
\pm \\
0.920\end{array}$ & $\begin{array}{c}36.348 \\
\pm \\
0.003\end{array}$ & $\begin{array}{c}0.433 \\
\pm \\
0.014\end{array}$ & $\begin{array}{c}0.057 \\
\pm \\
0.013\end{array}$ & $\begin{array}{c}0.008 \\
\pm \\
0.043\end{array}$ & $\begin{array}{c}0.084 \\
\pm \\
0.052\end{array}$ & $\begin{array}{c}2.010 \\
\pm \\
0.080\end{array}$ \\
\hline $\begin{array}{l}\text { Polluted soil from Marrow farms, } \\
\qquad 0 \mathrm{~cm}\end{array}$ & $\begin{array}{c}122.875 \\
\pm \\
0.003\end{array}$ & $\begin{array}{c}13.287 \\
\pm \\
0.124\end{array}$ & $\begin{array}{c}579.683 \\
\pm \\
0.001\end{array}$ & $\begin{array}{c}18.219 \\
\pm \\
0.004\end{array}$ & $\begin{array}{c}33.246 \\
\pm \\
0.035\end{array}$ & $\begin{array}{c}12.585 \\
\pm \\
0.015\end{array}$ & $\begin{array}{c}3.894 \\
\pm \\
0.437\end{array}$ & $\begin{array}{c}17.703 \\
\pm \\
0.026\end{array}$ \\
\hline $\begin{array}{l}\text { Polluted soil from Marrow farms, } \\
\qquad 0-30 \mathrm{~cm}\end{array}$ & $\begin{array}{c}264.692 \\
\pm \\
0.004\end{array}$ & $\begin{array}{c}12.959 \\
\pm \\
0.164\end{array}$ & $\begin{array}{c}1099.454 \\
\pm \\
0.005\end{array}$ & $\begin{array}{c}24.917 \\
\pm \\
0.009\end{array}$ & $\begin{array}{c}27.243 \\
\pm \\
0.002\end{array}$ & $\begin{array}{c}8.427 \\
\pm \\
0.043\end{array}$ & $\begin{array}{c}8.748 \\
\pm \\
0.148\end{array}$ & $\begin{array}{c}13.219 \\
\pm \\
0.083\end{array}$ \\
\hline $\begin{array}{l}\text { Clean soil from Marrow farms, } \\
\qquad 0 \mathrm{~cm}\end{array}$ & $\begin{array}{c}21.703 \\
\pm \\
0.002\end{array}$ & $\begin{array}{c}1.777 \\
\pm \\
0.010\end{array}$ & $\begin{array}{c}105.889 \\
\pm \\
0.002\end{array}$ & $\begin{array}{c}10.794 \\
\pm \\
0.004\end{array}$ & $\begin{array}{c}0.182 \\
\pm \\
0.009\end{array}$ & $\begin{array}{c}0.026 \\
\pm \\
0.029\end{array}$ & $\begin{array}{c}0.068 \\
\pm \\
0.101\end{array}$ & $\begin{array}{c}4.212 \\
\pm \\
0.010\end{array}$ \\
\hline $\begin{array}{l}\text { Clean soil from Marrow farms, } \\
\qquad 0-30 \mathrm{~cm}\end{array}$ & $\begin{array}{c}12.839 \\
\pm \\
0.001\end{array}$ & $\begin{array}{c}2.320 \\
\pm \\
0.011\end{array}$ & $\begin{array}{c}81.853 \\
\pm \\
0.190\end{array}$ & $\begin{array}{c}4.606 \\
\pm \\
0.012\end{array}$ & $\begin{array}{c}0.096 \\
\pm \\
0.008\end{array}$ & $\begin{array}{c}0.016 \\
\pm \\
0.071\end{array}$ & $\begin{array}{c}0.135 \\
\pm \\
0.119\end{array}$ & $\begin{array}{c}2.680 \\
\pm \\
0.120\end{array}$ \\
\hline FAO/WHO limit (mg/kg) & 100 & 5 & 437 & 10 & 6 & 0.2 & 2 & 10 \\
\hline
\end{tabular}

\subsection{Water Sampling}

Samples were collected and stored in clean plastic bottles and brought to the laboratory for analysis. Water samples were collected from the wells and the pools that were used for irrigating these vegetables in this region following the reported procedure [18].

\subsection{ICP-MS Analysis}

\subsubsection{Soil Analysis}

$80 \mathrm{ml}$ of milli-Q water were added to 20 grams of the soil sample. The soil samples were leaved for fourteen days. Then $2 \mathrm{ml}$ of the leachate were taken for analysis [18]. The samples were then analysed for the following metals $\mathrm{Ba}, \mathrm{Cu}$, $\mathrm{Pb}$, Th, Se, Mn, Co and As by ICP/MS (Agilent technologies 7500 series). For accurate quantitative determination of heavy metals in water samples, an internal standard method was used using $\mathrm{Nd}$ as internal standard and a multi-standard calibration method: 22 metals standard (Ag $10 \mathrm{mg} / \mathrm{L}, \mathrm{Al} 50 \mathrm{mg} / \mathrm{L}, \mathrm{B} 50 \mathrm{mg} / \mathrm{L}, \mathrm{Ba}$ 10 mg/L, Bi 100 mg/L, Ca 10 mg/L, Cd 10 mg/L, Co 10 mg/L, Cr 50 mg/L, Cu 10 mg/L, Fe 10 mg/L, K 100 mg/L, Li 50 mg/L, Mg 10 mg/L, Mn 10 mg/L, Mo 50 mg/L, Na 50 mg/L, Ni 50 mg/L, Pb 100 mg/L, Sr 10 mg/L, Tl 50 mg/L, Zn 10 $\mathrm{mg} / \mathrm{L}$, matrix $5 \% \mathrm{HNO}_{3}$ ). Samples were prepared by dilution of $1.0 \mathrm{~mL}$ of the water samples to $10.0 \mathrm{~mL}$ with $0.3 \%$ ultrapure nitric acid and analysed by ICP/MS. Each sample was analysed three times and the results are expressed as mean \pm SD (SD: standard deviation). Relative standard deviation (RSD) of the three results are calculated and found to be less than $5 \%$ for all samples for all 
metals analyzed in this study, reflecting the precision the method for the analysis of these heavy metals. Calibration curves for all metals analysed were constructed by plotting the ratio of the intensity of the analyse metal to that of the internal standard $(\mathrm{Nd})$ vs. concentration of the trace metal (in $\mu \mathrm{g} / \mathrm{L}$ ), and results showed that the calibration curves are linear with correlation coefficient $\left(\mathrm{r}^{2}\right)$ greater than 0.999 for the trace metals analysed [18].

\subsubsection{Vegetables Analysis}

The collected vegetable samples were washed with distilled water to remove dust particles. The samples were cut into small pieces. The vegetables' part was take, and dried in an oven at $50^{\circ} \mathrm{C}$. After drying, the samples were ready for acid digestion. 0.5 grams of the dried vegetable was digested with $5 \mathrm{ml}$ of $65 \%$ pure nitric acid using Mars 6 digestive apparatus, until the solution has transparent color. The digested samples were filtered using CA sterile syringe filters that has a diameter of $30 \mathrm{~mm}$ and the pore size $0.22 \mu \mathrm{m}$ [19]. Determination of heavy metals in the filtrate of vegetables was achieved using ICP-MS, as described above.

\subsubsection{Water Analysis}

Water samples were tested for their content by the addition of $65 \%$ pure nitric acid. $2 \mathrm{ml}$ of the sample were taken for analysis. They were analyzed by the use of ICP-MS [18].

\subsection{4. pH Soil Analysis}

The $\mathrm{pH}$ of soil samples was measured after the soil was soaked in water for more than $24 \mathrm{~h}$. $\mathrm{pH}$ of the filtrate was measured using a $\mathrm{pH}$ meter.

\section{Result and Discussion}

\subsection{Field Soil Samples}

The concentrations of heavy metals in soil samples are shown in Table 1. Lead concentrations for soils from $0 \mathrm{~cm}$ were ranged from $4.020 \mathrm{mg} / \mathrm{kg}$ to 5.344 $\mathrm{mg} / \mathrm{kg}$. Lead concentrations for samples taken from $0-30 \mathrm{~cm}$ mixture were ranged from $2.010 \mathrm{mg} / \mathrm{kg}$ to $5.360 \mathrm{mg} / \mathrm{kg}$. Lead concentrations in Corn soil, Bell pepper soil, Eggplant soil, Cucumber soil and Marrow soil at $0 \mathrm{~cm}$ were 12.211 $\mathrm{mg} / \mathrm{kg}, 34.144 \mathrm{mg} / \mathrm{kg}, 12.651,21.310 \mathrm{mg} / \mathrm{kg}$ and $17.703 \mathrm{mg} / \mathrm{kg}$ respectively. Lead concentrations for the $0-30 \mathrm{~cm}$ soil samples were $24.106 \mathrm{mg} / \mathrm{kg}, 22.915 \mathrm{mg} / \mathrm{kg}$, $10.216 \mathrm{mg} / \mathrm{kg}, 14.402 \mathrm{mg} / \mathrm{kg}$, and $13.219 \mathrm{mg} / \mathrm{kg}$ respectively. Lead concentrations in all samples were very high and exceed the limit given by WHO which is $10 \mathrm{mg} / \mathrm{kg}$ [13]. Lead is widely used in batteries and many industries. It is clear that the compost does not come from household sources, but it comes from sources that contain high concentration of lead [20]. Lead concentrations for all soil samples from clean soil farms did not exceed the limits given by FAO/WHO [13] (Table 1).

Barium concentrations in soil samples (Table 1 ) for the $0 \mathrm{~cm}$ soils for Corn soil, Bell pepper soil, Eggplant soil, Cucumber soil and Marrow soil were 175.962 
$\mathrm{mg} / \mathrm{kg}, 151.793 \mathrm{mg} / \mathrm{kg}, 125.384 \mathrm{mg} / \mathrm{kg}, 103.193 \mathrm{mg} / \mathrm{kg}$ and $122.875 \mathrm{mg} / \mathrm{kg} \mathrm{respec}-$ tively. Barium concentration for $0-30 \mathrm{~cm}$ mixture soil samples were 230.295 $\mathrm{mg} / \mathrm{kg}, 136.639 \mathrm{mg} / \mathrm{kg}, 62.641 \mathrm{mg} / \mathrm{kg}, 70.178 ; 264.692 \mathrm{mg} / \mathrm{kg}$ respectively. Barium concentration in soil samples was very high and exceeds the limit given by $\mathrm{WHO} / \mathrm{FAO}$ of $100 \mathrm{mg} / \mathrm{kg}$ [13]. It is important to say UDC main source does not come from Animal or Agricultural sources, but it comes from other sources that contain high concentration of Barium. Barium compounds are important pigment industry and paints [21]. Barium is also used in optical glass, ceramics, glazed pottery, glassware and Motor oil detergents [22]. Barium concentrations for all soil reference field samples at $0 \mathrm{~cm}$ and $0-30 \mathrm{~cm}$ did not exceed the limits given by [13] (Table 1).

Thallium concentrations in soil samples for Corn soil, Bell pepper soil, Eggplant soil, Cucumber soil and Marrow soil for $0 \mathrm{~cm}$ soil samples were $1.926 \mathrm{mg} / \mathrm{kg}$, $22.042 \mathrm{mg} / \mathrm{kg}, 23.200 \mathrm{mg} / \mathrm{kg}, 24.932 \mathrm{mg} / \mathrm{kg}$ and $13.287 \mathrm{mg} / \mathrm{kg}$ respectively. Thallium concentrations in soil samples at $0-30 \mathrm{~cm}$ soil mixture were $12.910 \mathrm{mg} / \mathrm{kg}$, $11.600 \mathrm{mg} / \mathrm{kg}, 16.020 \mathrm{mg} / \mathrm{kg}, 14.243 \mathrm{mg} / \mathrm{kg}$ and $12.959 \mathrm{mg} / \mathrm{kg}$ respectively. These concentrations exceed the limit given by WHO/FAO of $5 \mathrm{mg} / \mathrm{kg}$ [13]. Thallium is used in optics, high-density glasses electronics industry and radiation devices [23]. The concentration of Thallium in the clean farms at $0 \mathrm{~cm}$ and $0-30 \mathrm{~cm}$ soil mixtures does not exceed the FAO/WHO limit of $5 \mathrm{mg} / \mathrm{kg}$ [13].

Copper concentrations in soil samples for the soil farms at $0 \mathrm{~cm}$ was 17.656 $\mathrm{mg} / \mathrm{kg} ; 12.342 \mathrm{mg} / \mathrm{kg}, 13.709 \mathrm{mg} / \mathrm{kg}, 10.766 \mathrm{mg} / \mathrm{kg}, 33.246 \mathrm{mg} / \mathrm{kg}$, respectively. Copper concentrations for the 0 - $30 \mathrm{~cm}$ soil mixture were $34.870 \mathrm{mg} / \mathrm{kg}, 10.112$ $\mathrm{mg} / \mathrm{kg}, 7.110 \mathrm{mg} / \mathrm{kg}, 9.000 \mathrm{mg} / \mathrm{kg}$ and $27.243 \mathrm{mg} / \mathrm{kg}$ respectively (higher than WHO limit of $6 \mathrm{mg} / \mathrm{kg}$ [13] [23] (Table 1). Copper is used in wires, roofing and plumbing and pipe products (Callister, 2013). The value of copper in clean soil at $0 \mathrm{~cm}$ and $0-30 \mathrm{~cm}$ were very lower than WHO limits for all samples [24].

Manganese concentrations in soil samples (Table 1) at $0 \mathrm{~cm}$ were 741.838 $\mathrm{mg} / \mathrm{kg}, 951.016 \mathrm{mg} / \mathrm{kg}, 572.315 \mathrm{mg} / \mathrm{kg}$ and $579.683 \mathrm{mg} / \mathrm{kg}$ respectively. Manganese concentrations in soil samples at $0-30 \mathrm{~cm}$ soil mixtures were 1075.468 $\mathrm{mg} / \mathrm{kg}, 744.514 \mathrm{mg} / \mathrm{kg}, 545.220 \mathrm{mg} / \mathrm{kg}$ and $1099.454 \mathrm{mg} / \mathrm{kg}$ respectively. The concentration of Manganese exceeds the permissible limit set by WHO/FAO of $437 \mathrm{mg} / \mathrm{kg}$ [13]. Manganese is used in alloying, dry cells and paints industry [13] [25]. It is used in quantitative analysis techniques and Medicine. The concentrations of Manganese in the clean soil farm were below the standard limits for all samples [26].

Selenium concentration in soil samples (Table 1 ) were $5.860 \mathrm{mg} / \mathrm{kg} ; 12.871$ $\mathrm{mg} / \mathrm{kg} ; 3.621 \mathrm{mg} / \mathrm{kg} ; 7.600 \mathrm{mg} / \mathrm{kg}$ and $3.894 \mathrm{mg} / \mathrm{kg}$ respectively. For the $0-30$ $\mathrm{cm}$ mixture soils were $10.497 \mathrm{mg} / \mathrm{kg} ; 10.536 \mathrm{mg} / \mathrm{kg} ; 2.781 \mathrm{mg} / \mathrm{kg} ; 5.056 \mathrm{mg} / \mathrm{kg}$ and $8.748 \mathrm{mg} / \mathrm{kg}$ ), respectively. These concentrations are higher than FAO/WHO of $2 \mathrm{mg} / \mathrm{kg}$ [13]. Selenium is used in photovoltaic photocells, light meters and solar, photoconductive industry and glass industry [27]. The concentrations of Selenium in the clean soil farm were below the standard limits for all samples. 
Cobalt concentrations in soil samples at $0 \mathrm{~cm}$ were $17.934 \mathrm{mg} / \mathrm{kg}, 19.56 \mathrm{mg} /$ $\mathrm{kg}, 26.062 \mathrm{mg} / \mathrm{kg} ; 11.019 \mathrm{mg} / \mathrm{kg}, 18.219 \mathrm{mg} / \mathrm{kg}$ respectively. For the $0-30 \mathrm{~cm}$ soil samples the concentrations were $27.315 \mathrm{mg} / \mathrm{kg}, 12.197 \mathrm{mg} / \mathrm{kg}, 14.655 \mathrm{mg} / \mathrm{kg}$, $7.943 \mathrm{mg} / \mathrm{kg}$ and $24.917 \mathrm{mg} / \mathrm{kg}$, respectively. The concentrations are higher than WHO/FAO limits of $5 \mathrm{mg} / / \mathrm{kg}$ [13]. Cobalt has many applications in industry [28]. The concentrations of Cobalt in the clean soil farm were below the standard limits for all samples.

Arsenic concentrations in soil samples at $0 \mathrm{~cm}$ were $2.384 \mathrm{mg} / \mathrm{kg}, 5.172 \mathrm{mg} /$ $\mathrm{kg}, 6.362 \mathrm{mg} / \mathrm{kg}, 3.423 \mathrm{mg} / \mathrm{kg}$ and $12.58 \mathrm{mg} / \mathrm{kg}$ respectively. Arsenic concentrations in soil from $0-30 \mathrm{~cm}$ wer $3.360 \mathrm{mg} / \mathrm{kg}, 5.237 \mathrm{mg} / \mathrm{kg}, 3.264 \mathrm{mg} / \mathrm{kg}, 1.573$ $\mathrm{mg} / \mathrm{kg}$ and $8.427 \mathrm{mg} / \mathrm{kg}$, respectively. Arsenic concentrations are higher than $\mathrm{WHO} / \mathrm{FAO}$ limit of $0.2 \mathrm{mg} / \mathrm{kg}$ [13]. Arsenic is used in semiconductor and microchip industry. The concentrations of Arsenic in the clean soil farm were below the standard limits for all samples.

\subsection{Heavy Metals Concentration in Vegetable Samples}

The concentrations of heavy metals in vegetable samples are shown in Table 2. Lead concentrations in Corn soil, Bell pepper, Eggplant, Cucumber and Marrow that were fertilized by the compost were $0.900 \mathrm{mg} / \mathrm{kg}, 0.530 \mathrm{mg} / \mathrm{kg} ; 1 \mathrm{mg} / \mathrm{kg}$, $0.453 \mathrm{mg} / \mathrm{kg}$ and $0.549 \mathrm{mg} / \mathrm{kg}$, respectively. Lead concentrations in vegetables exceeded the WHO limit of $0.3 \mathrm{mg} / \mathrm{kg}$ [13] [29]. Lead concentrations in Corn soil, Bell pepper, Eggplant, Cucumber and Marrow in clean farms were 0.070 $\mathrm{mg} / \mathrm{kg}, 0.091 \mathrm{mg} / \mathrm{kg}, 0.035 \mathrm{mg} / \mathrm{kg}, 0.089 \mathrm{mg} / \mathrm{kg}$ and $0.084 \mathrm{mg} / \mathrm{kg}$. These concentrations are below the permissible limits. The concentrations of Barium in the vegetables were $1.398 \mathrm{mg} / \mathrm{kg}, 1.855 \mathrm{mg} / \mathrm{kg}, 3.021 \mathrm{mg} / \mathrm{kg}, 0.850 \mathrm{mg} / \mathrm{kg}$ and 2.641 $\mathrm{mg} / \mathrm{kg}$ respectively. Barium concentrations in these vegetables exceeded the WHO limit of $0.850 \mathrm{mg} / \mathrm{kg}$ [13]. Barium concentrations in the vegetables that were grown in the clean farms were below the permissible limits (Table 2). The concentrations of Thallium in the vegetables that were fertilized by the compost were $2.350 \mathrm{mg} / \mathrm{kg}, 1.215 \mathrm{mg} / \mathrm{kg}, 2.608 \mathrm{mg} / \mathrm{kg}, 1.608 \mathrm{mg} / \mathrm{kg}$ and $2.986 \mathrm{mg} / \mathrm{kg}$ respectively. Thallium concentrations in vegetables exceeded the WHO limit of 0.3 $\mathrm{mg} / \mathrm{kg}$ [13]. Thallium concentrations in the vegetables that were grown in the clean farms were below the permissible limits. The concentrations of Copper in the Corn, Bell pepper, Cucumber and Marrow vegetables that were fertilized by the compost were $59.586 \mathrm{mg} / \mathrm{kg}, 56.772 \mathrm{mg} / \mathrm{kg}, 50.527 \mathrm{mg} / \mathrm{kg}, 63.842 \mathrm{mg} / \mathrm{kg}$ respectively. Copper concentrations in these four vegetables exceeded the WHO limit of $40 \mathrm{mg} / \mathrm{kg}$ [13]. Copper concentration in the Eggplant sample was 23.049 $\mathrm{mg} / \mathrm{kg}$ below the permissible limits. Copper concentrations in the vegetables that were grown in the clean farms were below the permissible limits (Table 2). Manganese concentrations in Corn soil, Bell pepper, Eggplant, Cucumber and Marrow that were fertilized by the compost were $825.301 \mathrm{mg} / \mathrm{kg}, 463.743 \mathrm{mg} / \mathrm{kg}$, $795.835 \mathrm{mg} / \mathrm{kg}, 499.792 \mathrm{mg} / \mathrm{kg}$, and $446.196 \mathrm{mg} / \mathrm{kg}$. Manganese concentrations in these vegetables are very close or even exceeded the WHO limit of $500 \mathrm{mg} / \mathrm{kg}$ [13] [30]. Manganese concentrations in the vegetables that were grown in the 
Table 2. Heavy metals concentration in vegetables samples.

\begin{tabular}{|c|c|c|c|c|c|c|c|c|}
\hline \multirow{2}{*}{ Vegetables type } & \multicolumn{8}{|c|}{ Metals Concentration (mg/kg) } \\
\hline & $\mathrm{Ba}$ & $\mathrm{Tl}$ & $\mathrm{Mn}$ & Co & $\mathrm{Cu}$ & As & $\mathrm{Se}$ & $\mathrm{Pb}$ \\
\hline \multirow{3}{*}{ Corn from polluted farm } & 1.398 & 2.350 & 825.301 & 0.204 & 59.586 & 0.662 & 0.550 & 0.900 \\
\hline & \pm & \pm & \pm & \pm & \pm & \pm & \pm & \pm \\
\hline & 0.001 & 0.009 & 0.001 & 0.012 & 0.001 & 0.006 & 0.064 & 0.001 \\
\hline \multirow{3}{*}{ Corn from clean farm } & 0.133 & 0.154 & 21.114 & 0.082 & 2.493 & 0.162 & 0.061 & 0.070 \\
\hline & \pm & \pm & \pm & \pm & \pm & \pm & \pm & \pm \\
\hline & 0.001 & 0.022 & 0.012 & 0.001 & 0.003 & 0.017 & 0.015 & 0.001 \\
\hline \multirow{3}{*}{ Bell pepper from polluted farm } & 1.855 & 1.215 & 463.743 & 0.537 & 56.772 & 2.677 & 0.408 & 0.530 \\
\hline & \pm & \pm & \pm & \pm & \pm & \pm & \pm & \pm \\
\hline & 0.002 & 0.009 & 0.001 & 0.012 & 0.001 & 0.018 & 0.050 & 0.002 \\
\hline \multirow{3}{*}{ Bell pepper from clean farm } & 0.321 & 0.081 & 30.276 & 0.027 & 1.540 & 0.103 & 0.014 & 0.091 \\
\hline & \pm & \pm & \pm & \pm & \pm & \pm & \pm & \pm \\
\hline & 0.001 & 0.017 & 0.001 & 0.013 & 0.002 & 0.017 & 0.126 & 0.001 \\
\hline \multirow{3}{*}{ Eggplant from polluted farm } & 3.021 & 2.608 & 795.835 & 0.917 & 23.049 & 4.306 & 0.348 & \multirow{3}{*}{$\begin{array}{r}1.000 \\
\pm 0.003\end{array}$} \\
\hline & \pm & \pm & \pm & \pm & \pm & \pm & \pm & \\
\hline & 0.003 & 0.012 & 0.025 & 0.009 & 0.002 & 0.003 & 0.114 & \\
\hline \multirow{3}{*}{ Eggplant from clean farm } & 0.226 & 0.139 & 46.808 & 0.068 & 6.245 & 0.049 & 0.076 & \multirow{3}{*}{$\begin{array}{c}0.035 \\
\pm 0.004\end{array}$} \\
\hline & \pm & \pm & \pm & \pm & \pm & \pm & \pm & \\
\hline & 0.004 & 0.032 & 0.017 & 0.007 & 0.001 & 0.024 & 0.015 & \\
\hline \multirow{3}{*}{ Cucumber from polluted farm } & 0.850 & 1.608 & 499.792 & 0.522 & 50.527 & 1.591 & 0.417 & \multirow{3}{*}{$\begin{array}{c}0.453 \\
\pm 0.006\end{array}$} \\
\hline & \pm & \pm & \pm & \pm & \pm & \pm & \pm & \\
\hline & 0.006 & 0.033 & 0.005 & 0.003 & 0.001 & 0.023 & 0.041 & \\
\hline \multirow{3}{*}{ Cucumber from clean farm } & 0.253 & 0.250 & 88.271 & 0.083 & 7.366 & 0.113 & 0.056 & 0.089 \\
\hline & \pm & \pm & \pm & \pm & \pm & \pm & \pm & \pm \\
\hline & 0.001 & 0.031 & 0.001 & 0.007 & 0.001 & 0.008 & 0.038 & 0.001 \\
\hline \multirow{3}{*}{ Marrow from polluted farm } & 2.641 & 2.986 & 446.196 & 1.119 & 63.842 & 3.798 & 0.348 & 0.549 \\
\hline & \pm & \pm & \pm & \pm & \pm & \pm & \pm & \pm \\
\hline & 0.003 & 0.006 & 0.001 & 0.004 & 0.002 & 0.015 & 0.108 & 0.003 \\
\hline \multirow{3}{*}{ Marrow from clean farm } & 0.073 & 0.160 & 3.154 & 0.057 & 5.487 & 0.088 & 0.028 & 0.084 \\
\hline & \pm & \pm & \pm & \pm & \pm & \pm & \pm & \pm \\
\hline & 0.002 & 0.021 & 0.001 & 0.001 & 0.035 & 0.012 & 0.032 & 0.002 \\
\hline FAO/WHO limit (mg/kg) & 0.85 & 0.3 & 500 & 0.1 & 40 & 0.2 & 0.3 & 0.3 \\
\hline
\end{tabular}

clean farms were below the permissible limits (Table 2). The concentrations of Selenium in the vegetables that were fertilized by the compost were $0.550 \mathrm{mg} / \mathrm{kg}$, $0.408 \mathrm{mg} / \mathrm{kg}, 0.348 \mathrm{mg} / \mathrm{kg}, 0.417 \mathrm{mg} / \mathrm{kg}$ and $0.348 \mathrm{mg} / \mathrm{kg}$ respectively. Selenium concentrations in vegetables exceeded the WHO limit of $0.3 \mathrm{mg} / \mathrm{kg}$ [13]. Selenium concentrations in the vegetables that were grown in the clean farms were below the permissible limits. The concentrations of Cobalt in Eggplant, Bell pepper, Cucumber and Marrow vegetables that were fertilized by the compost were $0.537 \mathrm{mg} / \mathrm{kg}, 0.917 \mathrm{mg} / \mathrm{kg}, 0.522 \mathrm{mg} / \mathrm{kg}, 1.119 \mathrm{mg} / \mathrm{kg}$ respectively. Cobalt concentrations in these four vegetables exceeded the WHO limit of $0.1 \mathrm{mg} / \mathrm{kg}$ [31] [32]. Cobalt concentrations in the Corn samples were below the permissible 
limits. Cobalt concentrations in the vegetables that were grown in the clean farms were also below the permissible limits (Table 2). Arsenic concentrations in Corn soil, Bell pepper, Eggplant, Cucumber and Marrow vegetables that were fertilized by the compost were $0.662 \mathrm{mg} / \mathrm{kg}, 2.677 \mathrm{mg} / \mathrm{kg}, 4.306 \mathrm{mg} / \mathrm{kg}, 1.591$ $\mathrm{mg} / \mathrm{kg}, 3.798 \mathrm{mg} / \mathrm{kg}$, respectively. Arsenic concentrations in these vegetables exceeded the WHO limit of $0.2 \mathrm{mg} / \mathrm{kg}$ [13] [31] [32]. Arsenic concentrations in the vegetables that were grown in the clean farms were below the permissible limits (Table 2)

\subsection{Heavy Metals Concentration in Water Used for Irrigation}

The concentration of lead, cobalt, copper, Arsenic, Selenium, Barium in Agricultural water Pools were $0.042 \mathrm{mg} / \mathrm{kg}, 0.118 \mathrm{mg} / \mathrm{kg}, 0.163 \mathrm{mg} / \mathrm{kg}, 0.051 \mathrm{mg} / \mathrm{kg}$, $\mathrm{mg} / \mathrm{kg}, 0.052 \mathrm{mg} / \mathrm{kg}$ and $0.317 \mathrm{mg} / \mathrm{kg}$ respectively. The concentration of these metals in the Wells were $0.064 \mathrm{mg} / \mathrm{kg} ; 0.034 \mathrm{mg} / \mathrm{kg}, 0.062 \mathrm{mg} / \mathrm{kg}, 0.042,0.047$ $\mathrm{mg} / \mathrm{kg}$ and $0.307 \mathrm{mg} / \mathrm{kg}$ respectively). All of these values are below the WHO limit of $2.236 \mathrm{mg} / \mathrm{kg}, 0.224 \mathrm{mg} / \mathrm{kg}, 0.447 \mathrm{mg} / \mathrm{kg}, 0.316 \mathrm{mg} / \mathrm{kg}, 0.141 \mathrm{mg} / \mathrm{kg}, 1$ $\mathrm{mg} / \mathrm{kg}$ for lead, cobalt, copper, Arsenic, Selenium and Barium respectively [18]. Thalium metal was not detected in this water. Manganese was approximately equal to WHO limit of 0.447 .

\section{4. pH of Soil Samples}

The $\mathrm{pH}$ of soil samples ranges between 7.15 and 8.05. There is no clear relation between compost farms and $\mathrm{pH}$ values. Soil $\mathrm{pH}$ was basic for all clean and compost soil farms.

\section{Conclusion}

From the present study, it can be concluded that heavy metals concentrations in soil and vegetables are very high when compost is applied as a fertilizer. Heavy metals concentrations are above the recommended levels of the WHO/FAO limit. Clearly, the compost is largely composed of industrial wastes. This kind of compost should not be used as fertilizer because of its high content of heavy metals.

\section{Conflicts of Interest}

The authors declare no conflicts of interest regarding the publication of this paper.

\section{References}

[1] Choudhury, T.R. (2015) Heavy Metals Contamination in Vegetables and Its Growing Soil. International Journal of Environmental Analytical Chemistry, 2, 6.

[2] Khalilia, W. (2020) Assessment of Lead, Zinc and Cadmium Contamination in the Fruit of Palestinian Date Palm Cultivars Growing at Jericho Governorate. Journal of Biology, Agriculture and Healthcare, 10, 7-14.

[3] Bradl, H. (2002) Heavy Metals in the Environment: Origin, Interaction and Remediation. Academic Press, London. 
[4] Alengebawy, A., Abdelkhalek, S.T., Qureshi, S.R. and Wang, M.Q. (2021) Heavy Metals and Pesticides Toxicity in Agricultural Soil and Plants: Ecological Risks and Human Health Implications. Toxics, 9, Article No. 42. https://doi.org/10.3390/toxics9030042

[5] Kabata-Pendias, A. (2000) Trace Elements in Soils and Plants. 3rd Edition, CRC Press, Boca Raton. https://doi.org/10.1201/9781420039900

[6] Palestinian Ministry of Agriculture (2016) Technical Guide to Preparing Compost. Ramallah.

[7] TMECC (2000) Test Method for Examination of Composts and Composting. United States Composting Council.

[8] Zennaro, M., Cristofori, F., Formigoni, D., Frignani, F. and Pavoni, B. (2005) Heavy Metal Contamination in Compost. A Possible Solution. Annali di Chimica, 95, 247-256. https://doi.org/10.1002/adic.200590027

[9] Toledo, M., Siles, J., Gutiérrez, M. and Martín, M. (2018) Monitoring of the Composting Process of Different Agroindustrial Waste: Influence of the Operational Variables on the Odorous Impact. Waste Management, 76, 266-274.

[10] Isaac, J., Khair, A., Hilal, J., et al. (2011) Status of the Environment in the Occupied Palestinian Territory, a Human Rights-Based Approach. The Applied Research Institute Jerusalem (ARIJ), Bethlehem.

[11] Isaac, J., Salem, S.S., Hilal, J., et al. (2007) Status of the Environment in the Occupied Palestinian Territory. Applied Research Institute Jerusalem (ARIJ), Bethlehem.

[12] Center Palestinian National Information (2011) Impact of the Israeli Colonies on the Palestinian Environment. Palestinian News and Info Agency.

http://info.wafa.ps/atemplate.aspx?id=4073\#

[13] FAO/WHO (2011) Joint FAO/WHO Food Standards Programme Codex Committee on Contaminants in Foods. 64-89.

[14] Mohod, C.V. (2015) A Review on the Concentration of the Heavy Metals in Vegetable Samples Like Spinach and Tomato Grown Near the Area of Amba Nalla of Amravati City. International Journal of Innovative Research in Science, Engineering and Technology, 4, 2788-2792.

[15] The Applied Research Institute (2012) Al Jiftlik Village Profile. http://vprofile.arij.org/jericho/pdfs/vprofile/Al\%20Jiftlik_en_FINAL.pdf

[16] Palestinian Central Bureau of Statistics (2016) Palestine in Figures. Ramallah.

[17] He, Z.L., Yang, X.E. and Stoffella, P.J. (2005) Trace Elements in Agroecosystems and Impacts on the Environment. Journal of Trace Elements in Medicine and Biology, 19, 125-140. https://doi.org/10.1016/j.jtemb.2005.02.010

[18] Malassa, H., Al-Qutob, M., Al-Khatib, M. and Al-Rimawi, F. (2013) Determination of Different Trace Heavy Metals in Ground Water of South West Bank/Palestine by ICP/MS. Journal of Environmental Protection, 4, 818-827. https://doi.org/10.4236/jep.2013.48096

[19] Aweng, E.R., Karimah, M. and Suhaimi, O. (2011) Heavy Metals Concentration of Irrigation Water, Soils and Fruit Vegetables in Kota Bharu Area, Kelantan, Malaysia. Journal of Applied Sciences in Environmental Sanitation, 6, 463-470.

[20] Zheng, N., Wang, Q.C. and Zheng, D.M. (2007) Health Risk of Hg, Pb, Cd, Zn, and $\mathrm{Cu}$ to the Inhabitants around Huludao Zinc Plant in China via Consumption of Vegetables. Science of the Total Environment, 383, 81-89. https://doi.org/10.1016/j.scitotenv.2007.05.002

[21] Attallah, N. (2020) Palestine: Solid Waste Management under Occupation. Heinrich Böll Stiftung, Palestine and Jordan. 
https://ps.boell.org/en/2020/10/07/palestine-solid-waste-management-under-occup ation

[22] Kresse, R., Baudis, U., Jäger, P., Riechers, H.H., Wagner, H., Winkler, J. and Wolf, H.U. (2007) Barium and Barium Compounds. Ullmann's Encyclopedia of Industrial Chemistry. https://doi.org/10.1002/14356007.a03_325.pub2

[23] Lide, D.R. (2003-2004) CRC Handbook of Chemistry and Physics. 84th Edition, CRC Press, Boca Raton.

[24] Sun, Q.B., Ying, C.Q. and Deng J.F. (2013) Characteristics of Soil-Vegetable Pollution of Heavy Metals and Health Risk Assessment in Daye Mining Area. Chemical Environment, 32, 671-677.

http://en.cnki.com.cn/Article_en/CJFDTOTAL-HJHX201304023.htm

[25] Stern, B.R. (2010) Essentiality and Toxicity in Copper Health Risk Assessment: Overview, Update and Regulatory Considerations. Journal of Toxicology and Environmental Health, Part $A, 73,114-127$. https://doi.org/10.1080/15287390903337100

[26] European Union (2006) Commission Regulation (EC) No. 1881/2006 of 19 December 2006 Setting Maximum Levels for Certain Contaminants in Foodstuffs. Official Journal of the European Union, 364, 5-24.

[27] Tchounwou, P.B., Yedjou, C.G., Patlolla, A.K. and Sutton, D.J. (2012) Heavy Metal Toxicity and the Environment. Molecular, Clinical and Environmental Toxicology, 101, 133-164.

[28] Irfan, N.M., Khan, I.N., Islam, S., et al. (2016) Presence of Heavy Metals in Fruits and Vegetables: Health Risk in Bangladesh. Chemosphere, 152, 431-438.

[29] Luo, C.L., Liu, C.P., Wang, Y., Liu, X., Li, F.B., Zhang, G. and Li, X.D. (2011) Heavy Metal Contamination in Soils and Vegetables near an E-Waste Processing Site, South China. Journal of Hazardous Materials, 186, 481-490.

https://doi.org/10.1016/j.jhazmat.2010.11.024

[30] McLaughlin, M.J., Smolders, E., Degryse F. and Rietra, R. (2011) Uptake of Metals from Soil into Vegetables. In: Swartjes, F., Ed., Dealing with Contaminated Sites. From Theory towards Practical Application, Springer, Dordrecht, 325-367. https://doi.org/10.1007/978-90-481-9757-6_8

[31] Chang C.Y., Yu J.J., Chen F.B., et al. (2013) Accumulation of Heavy Metals in Leaf Vegetables from Agricultural Soils and Associated Potential Health Risks in the Pearl River Delta, South China. Environmental Monitoring and Assessment, 186, 1547-1560.

[32] Bradl, H. (2005) Sources and Origins of Heavy Metals. In: Bradl, H., Ed., Heavy Metals in the Environment: Origin, Interaction and Remediation, Elsevier, Amsterdam. 